\title{
sciendo \\ The importance of prediction methods in industry 4.0 on the example of steel industry
}

doi:10.2478/mape-2019-0028

Date of submission to the Editor: 04/2018

Date of acceptance by the Editor: 07/2018

MAPE 2019, volume 2, issue 1, pp. 283-295

\section{Bożena Gajdzik}

ORCID ID: 0000-0002-0408-1691

Silesian University of Technology, Poland

\section{INTRODUCTION}

With the technological advancement, the industry concept in the $21^{\text {st }}$ century is referred to as Industry 4.0 (German: Industrie 4.0) - the equivalent of the fourth industrial revolution in connection with the use of automatic machines, autonomous robots and digital technology. Digital transformation is the most important trend in the global economy. Enterprises use IT technology and apply (more often) artificial intelligence. The essence of Industry 4.0 is to integrate the automation and robotization of production processes with systems and created networks and people. The basic structure of Industry 4.0 is made up of cyber-physical systems, the Internet of Things (IOT) and cloud computing. The realization of technology development is a smart factory in which cyber-physical systems (CPS) that control physical processes, create virtual (digital) copies of the real world and make decentralized decisions, and through the Internet of Things in real time communicate and cooperate with each other and with people, while through processing the internal and inter-operative services are offered and used (Bauernhansl et al., 2014; Gerbert et al., 2015; Schwab, 2016). The purpose of this new concept is higher (than before) efficiency and cost reduction, but also the speed of reaction to the ever-changing consumer needs and other dynamic phenomena in volatile markets. New business models are created on the market (Grabowska, 2016).

Companies that introduce new solutions to the fourth industrial revolution take over the market and customers, deepening their competitive edge. Industry sectors that are commencing changes at level 4.0 include the steel industry. In domestic conditions (territory coverage: Poland), the largest metallurgical enterprise - Arcelor Mittal Poland - introduces technological changes that will be building the foundations of intelligent production in the future. The potential production capacity of the company accounts for $70 \%$ of steel production in Poland (Gajdzik \& Sroka, 2012). The company belongs to the largest global capital group in the field of steel production volume on the global market - the Arcelor Mittal Group. The Group produced 97.03 million tonnes (Mt) crude steel in 2017 (World Steel Association report: Top steelmakers in 2017). With relatively large investment opportunities, ArcelorMittal's staffing and research facilities can act as a pioneer in the trail. However, at the current stage of change it is difficult to find an example of a comprehensive approach to the industrial

\footnotetext{
*bozena.gajdzik@polsı.pl
} 
revolution, to implement Industry 4.0 solutions simultaneously at all levels of the business structure. Changes are introduced sequentially in various (selected) business segments (implementation of partial solutions). The scope of these changes is described in the first part of the work: Steel production in Industry 4.0. This part of the work was based on a study of literature and observation of changes in the steel sector in Poland. Observation was conducted by the author of this publication. Changes in production in the perspective of further development of Industry 4.0 in aspects of both mass and personalized require the use of predictive methods to production plan and control maintenance. Thanks to sensors, algorithms, advanced analytics and the ability to draw conclusions from information, producers can more effectively control production. Digital technologies connect machines, products and teams, creating new opportunities, including virtual simulations, advanced analysis, spatial printing, remote expertise and real-time collaboration. Technologies used in Industry 4.0 combine reality with virtuality. As a result, the real-time monitoring system removes employers into a virtual image (space). Current information is compiled with perspective information. The dual system of device operation analysis gives the possibility of controlling the production process in the extended horizon: virtual or extended reality (VR/AR) (Jasperneite, 2012; Sendler, 2013).

The production prediction is based on the regularities characterizing the forecasted phenomenon and the dependencies between the various components of production (cause-and-effect relationships, similarities in development, symptomatic relationships between the forecast phenomenon and other phenomena) (StatSoft, 2012, Dittmann, 2011).

In the perspective of the in the latest technological solutions in steel production that are expected to bring metallurgical enterprises to the level of 4.0, a prospective analysis of the production volume is necessary. In part of the work: Forecasts of the world steel production summarized the forecasts prepared by the author of this publication. This combination of steel production forecast (Table 1) can be a valuable research material for the short- or medium-term planning of steel production by steelmaking companies with global and international reach.

\section{STEEL PRODUCTION IN INDUSTRY 4.0}

The changes implemented in manufacturing enterprises (steelmaking companies) aspiring to the role of leaders in Industry 4.0 are an alternative to the traditional development of the enterprise. Enterprises of individual industry sectors are currently at various stages of investment work progress, which are to lead companies to achieve the level 4.0 (Saniuk et al., 2013). Investment projects are most often implemented in a selected manufacturing process for a specific product or group of products within core business (Kagermann, et al., 2013).

Enterprises from various industry sectors implement pilot projects (start-up programs). Such programs are a form of gradual changes in production. Enterprises adopt a two-pronged production strategy, implementing and improving traditional production and investing in digital technological solutions that are to lead to the creation of autonomous cyber-physical production systems - CPPS. Such a way of development of companies allows them to produce and sell on the one hand as it has been so far (not every production profile requires installation of robots), and on the other hand they can monitor, modify and service real-time production equipment. 
Stock market investors more and more often pay attention not to material resources (factories, machines) but to innovation, technology and knowledge. There are industries on the market where the scope of implemented changes on the way to smart production is larger (wider) than in other industries, eg automotive sector. In industries belonging to heavy industry: mining and metallurgy, innovations are more traditional.

ArcelorMittal Poland invests in advanced technologies and in computer software production and related processes. Investment works are carried out on individual devices, obtaining computer control of their work (exemplary devices: blast furnace, converters, plastic processing equipment) and on selected metallurgical products obtained using the latest technological advancements, eg modeling of 3D products. Thanks to sensors, algorithms, advanced analytics and the ability to draw conclusions from information, production companies can more effectively eliminate bottlenecks in production. Data from devices are gathered in one place - data center. The existing IT and computer systems implemented in metallurgical enterprises, eg ERP, SAP are expanded and adapted to the requirements of intelligent production control (software customization). Examples of investments carried out at ArcelorMittal Poland in recent years: modernization of a blast furnace in Cracow, refurbishment of heaters, a new blast furnace cooling system, hybrid filters in the sintering belt sintering system in Dąbrowa Górnicza (the first such installation in Poland). These investments are solutions outside the Industry 4.0, but significantly reducing the impact of steel production on the environment (lower water and coke consumption, reduction of emissions) (Special Report, 2018).

Investment implementations are part of the area of change known as "zero waste" and Lean Manufacturing (Grabowska, 2018; Furman et all., 2017) and higher efficiency in production (Gajdzik \& Galwik, 2017). In the last 30 years, steel production in Poland has changed radically, obsolete and uneconomical steel production technologies have been withdrawn, the level of work automation has increased and manual labor has been reduced (Gajdzik, 2013).

Currently, metallurgical enterprises (international capital groups) are seeing deeper automation and stronger integration of infrastructure and data. The process of digitization and computerization is primarily implemented in the area of production harmonization. Industry 4.0 also enters the sectors related to steel production. The industry in a particular way conditioned in the implementation of Industry 4.0 solutions is welding - welding robotics of individual elements in enterprises. Modern technology are used in industry branches (sectors) that are associated with rather less complicated production, such as: steel constructions, agricultural machinery, metal fences. In addition to production, changes are introduced in logistics - modern warehouses of steel products, equipped with automatic devices for registering and servicing orders - steel structure warehouse in Dąbrowa Górnicza belonging to Thyssen Energo Stal (Gajdzik, 2019; Kramarz, 2012).

An important area of change is also building new customer relationship. ArcelorMittal has launched the Steel Advisor for Industry platform. The platform is an online guide and helps customers find the right metallurgical product depending on the target application. Transformations in production have an impact on the labor market. Automation, robotization of works and artificial intelligence that is used in machines, robots and software is able to replace a human being. Industry 4.0 requires different 
competencies of employees in various areas of the organization. Currently, there is a growing demand for: automation and robotics engineers (Report PWC).

ArcelorMittal Poland has started recruitment for positions for the servicing of automated production lines, looking for engineers 4.0 (footnote). The existing organizational structures are radically changed by the creation and/or expansion of IT departments and the establishment of data analysis teams, as well as by cyberphysical production systems. Managers for Industry 4.0 are located at the top management level in this organization.

Summing up, at the current stage of development of metallurgical enterprises in Poland (taking into account the strong influence of foreign capital, which owns the largest steel mills), the automation of production processes is carried out, by using more and more modern machines, which does not exhaust the essence of Industry 4.0, but these are changes that lead to Industry 4.0. Metallurgical enterprises at the current stage of development, combine and integrate processes and devices with each other, deepening automation and robotization resulting from the previous industrial revolution (3.0) and using cloud computing technology to control production, as well as 3D printing for product design and presentation of market offers (Report $P W C)$. By 2020, the metallurgical industry is planning an annual increase in investment by $4 \%$ in the aforementioned work area (weighted average of $5 \%$ ) (Report, PWC). Investments in IT infrastructure will be developed systematically. Sensors will be installed on individual production machines, as well as solutions allowing to connect production devices to the network and business platforms (Sroka et al., 2014). New technology creates the intelligent production process with new products (Sitko, 2015) and increases the quality of standards of its. The problem of quality of products is discussed by scientists (Sitko et al., 2018; Gajdzik \& Sitko, 2014; Gajdzik \& Sitko, 2016).

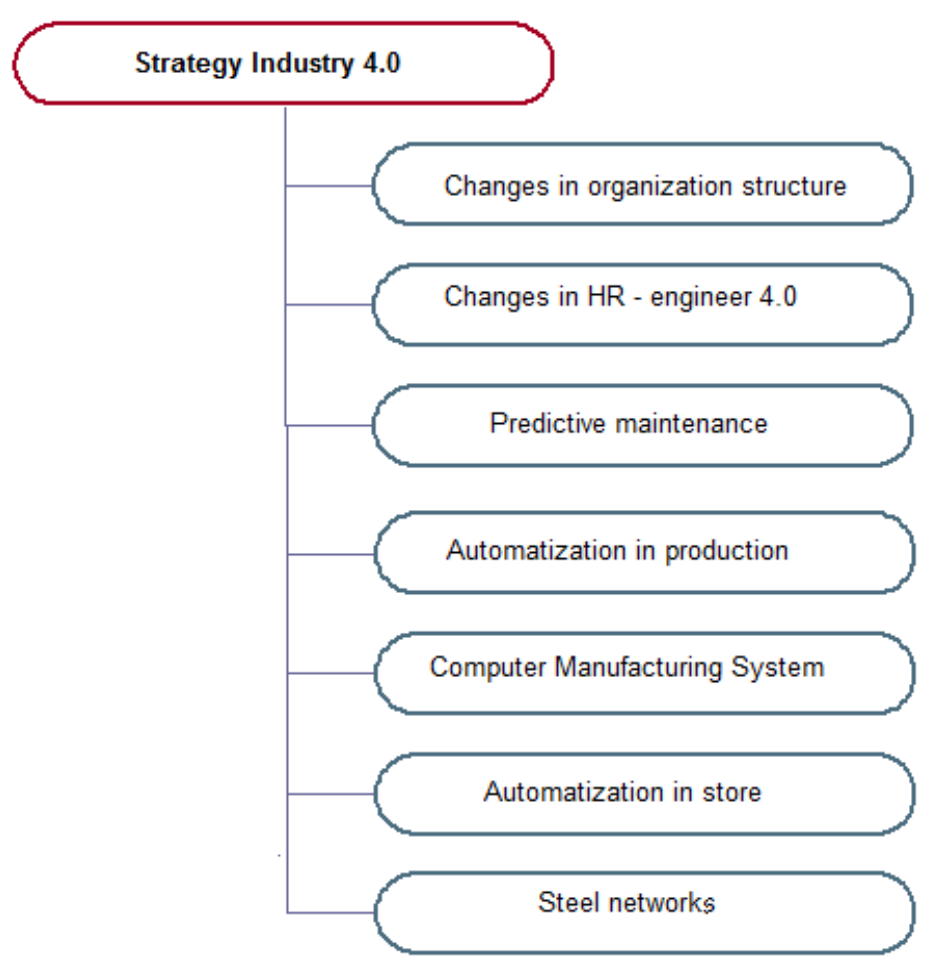

Fig. 1 Fields of changes in an steel enterprise in I 4.0 


\section{PREDICTION OF STEEL PRODUCTION IN INDUSTRY 4.0}

Forecasts are built on the basis of empirical data in order to obtain production in the future. At the stage of initiating changes in steel production under the pressure of the economy 4.0, the prediction (forecast) of steel production is used in the planning phase of production processes. Steel production forecasts provide information for planning machine load, material demand, determining the range of products and the number of employees. Thanks to production forecasts, technologists can, for example, test and optimize machine settings, harmonize production before starting it physically, shortening material ordering time, etc. Production forecasts are used to simulate production (simulations can use real-time data to reflect the physical world in a virtual model). Information on the course of steel production in metallurgical enterprises (the example of ArcelorMittal Poland) is organized into a vertical pyramid of automation, in which sensors and field devices with limited intelligence and automation controllers supply the superior control system of the production process. In the future, the sector will, like other industry sectors, strive to incorporate steel production technologies into the network (including the protection of critical industrial systems and production lines). Metallurgical enterprises from the data used on the Intranet of the company, while maintaining the principles of cyber security, will create a business platform and block chain.

\section{Forecasts of the world steel production}

In this part of the work, steel production forecasts (table1) have been compiled. The sources of empirical data are World Steel Association reports (Steel Statistical Yearbook). The forecasting methodology was implemented sequentially (Dittmann, 2016 pp. 25-33; Green, 2003; Snarska, 2005; Zeliaś, 1997) and included: 1. Formulating the research goal - scientific goal - popularizing built forecasts in the scientific community, 2. Indicating factors that influence the forecasted phenomenon - the global steel market situation (core factor), 3. Collection statistical processing and analysis of forecast data - current steel production trend with random fluctuations caused by the global economic crisis in 2008-2010, 4. Selection of forecasting methods - classic trend models and adaptive trend models (single-equation models), 5. Design of forecasts in the layout: total steel production, BOF steel production, EAF steel production, 6 . Assessment of the acceptability of the forecast, 7. Presentation of the obtained forecasts in scientific publications (in accordance with the adopted research goal), 8. Assessment of the accuracy of the forecast - opinions of experts from the steel industry, intuitive assessments, AHP method. This publication summarizes the obtained forecasts in the system: total production and according to technological processes. This structure of forecasts will allow managers to make production decisions, including in the area of introducing technological changes. In Poland, due to the high costs of environmental protection, management considers the decision to replace BOF technology by EAF. However, another problem arises because energy costs in the steel sector in Poland are much higher than in neighboring EU countries, eg Germany (even by approx. 60\%). 
Table 1

Forecasts of the world steel production

\begin{tabular}{|c|c|c|c|c|}
\hline No. & Method & $\begin{array}{l}\text { Total } \\
(\mathrm{Mt})\end{array}$ & $\begin{array}{l}\text { BOF } \\
\text { (Mt) }\end{array}$ & $\begin{array}{l}\text { EAF } \\
(\mathrm{Mt})\end{array}$ \\
\hline 1 & Additive naïve method (point forecast) & 1690.479 & 1206.963 & 471.778 \\
\hline 2 & $\begin{array}{l}\text { Multiplicative naïve method with increasing } \\
\text { tendency }\end{array}$ & $\begin{array}{l}\text { a) } 1756.430 \\
\text { b) } 1824.955 \\
\text { c) } 1896.152 \\
\text { d) } 1970.128 \\
\text { e) } 2046.128\end{array}$ & $\begin{array}{l}1214.192 \\
1221.464 \\
1228.780 \\
1236.140 \\
1243.544 \\
\end{array}$ & $\begin{array}{l}532.838 \\
601.789 \\
679.670 \\
767.629 \\
866.972\end{array}$ \\
\hline 3 & $\begin{array}{l}\text { Simple moving average for time series } \\
\text { with constant k-point value }(k=2)\end{array}$ & $\begin{array}{l}1658.742 \\
1674.610 \\
1666.676 \\
1670.643 \\
1668.659\end{array}$ & $\begin{array}{l}1203.370 \\
1205.167 \\
1204.268 \\
1204.717 \\
1204.493\end{array}$ & $\begin{array}{l}444.749 \\
458.263 \\
451.506 \\
454.885 \\
453.195\end{array}$ \\
\hline 4 & $\begin{array}{l}\text { Simple moving average for time series } \\
\text { with constant } \mathrm{k} \text {-point value }(k=3)\end{array}$ & $\begin{array}{l}1638.470 \\
1651.984 \\
1660.311 \\
1654.183 \\
1662.220\end{array}$ & $\begin{array}{l}1203.480 \\
1203.407 \\
1204.616 \\
1203.834 \\
1203.952\end{array}$ & $\begin{array}{l}426.149 \\
438.549 \\
445.492 \\
436.730 \\
440.257\end{array}$ \\
\hline $5 a$ & $\begin{array}{l}\text { Weighted moving average for time series } \\
\text { with constant k-point value }(k=2) \text { and weighs } \\
\qquad\left(w_{1}=0.40 ; w_{2}=0.60\right)\end{array}$ & $\begin{array}{l}1665.089 \\
1675.245 \\
1671.183 \\
1672.808 \\
1672.158\end{array}$ & $\begin{array}{l}1204.089 \\
1205.238 \\
1204.778 \\
1204.962 \\
1204.889\end{array}$ & $\begin{array}{l}450.154 \\
458.804 \\
455.344 \\
456.728 \\
456.174\end{array}$ \\
\hline $5 b$ & $\begin{array}{l}\text { Weighted moving average for time series } \\
\text { with constant k-point value }(k=2) \text { and weighs } \\
\qquad\left(w_{1}=0.30 ; w_{2}=0.70\right)\end{array}$ & $\begin{array}{l}1671.437 \\
1677.149 \\
1675.435 \\
1675.950 \\
1675.795\end{array}$ & $\begin{array}{l}1204.807 \\
1205.454 \\
1205.260 \\
1205.318 \\
1205.301\end{array}$ & $\begin{array}{l}455.560 \\
460.426 \\
458.966 \\
459.404 \\
459.273\end{array}$ \\
\hline 6 & $\begin{array}{l}\text { Weighted moving average for time series with } \\
\text { constant k-point value }(k=3) \text { and weighs } \\
\text { (for columns no. } 4 \text { and } 5: w_{1}=0.10 ; w_{2}=0.20 \text {; } \\
w_{2}=0.70 ; \text { for column no. } 3: w_{1}=0.10 ; w_{2}=0.30 \text {; } \\
\left.\qquad w_{2}=0.60\right)\end{array}$ & $\begin{array}{l}1662.181 \\
1667.153 \\
1667.994 \\
1667.160 \\
1667.410\end{array}$ & $\begin{array}{l}1205.199 \\
1205.010 \\
1205.243 \\
1205.192 \\
1205.184\end{array}$ & $\begin{array}{l}452.683 \\
453.006 \\
454.819 \\
454.242 \\
454.234\end{array}$ \\
\hline 7 & $\begin{array}{l}\text { Simple moving average for increasing time } \\
\text { series with } k \text {-point } k=2\end{array}$ & $\begin{array}{l}1736.756 \\
1791.631 \\
1842.207 \\
1894.933 \\
1946.584\end{array}$ & $\begin{array}{l}1208.595 \\
1213.004 \\
1216.025 \\
1219.740 \\
1223.108\end{array}$ & $\begin{array}{l}513.192 \\
560.929 \\
605.504 \\
651.661 \\
697.026\end{array}$ \\
\hline 8 & $\begin{array}{c}\text { Simple moving average for increasing time } \\
\text { series with k-point } k=3\end{array}$ & $\begin{array}{l}1698.254 \\
1731.697 \\
1766.594 \\
1791.966 \\
1823.203\end{array}$ & $\begin{array}{l}1199.509 \\
1198.112 \\
1197.558 \\
1194.422 \\
1192.727\end{array}$ & $\begin{array}{l}485.620 \\
516.844 \\
551.219 \\
577.699 \\
608.392\end{array}$ \\
\hline $9 a$ & $\begin{array}{l}\text { Weighted moving average for increasing time } \\
\text { series with k-point } k=3 \text { and weights (for } \\
\text { columns no. } 4 \text { and } 5: w_{1}=0.15 ; w_{2}=0.25 \\
w_{2}=0.60 ; \text { for column no. } 3: w_{1}=0.20 ; w_{2}=0.30 \\
\qquad w_{2}=0.50 \text { ) }\end{array}$ & $\begin{array}{l}1717.094 \\
1755.260 \\
1795.023 \\
1831.677 \\
1869.565\end{array}$ & $\begin{array}{l}1206.450 \\
1207.351 \\
1208.841 \\
1209.883 \\
1211.016\end{array}$ & $\begin{array}{l}505.211 \\
543.100 \\
582.301 \\
620.309 \\
658.597\end{array}$ \\
\hline $9 b$ & $\begin{array}{l}\text { Weighted moving average for increasing time } \\
\text { series with k-point } k=3 \text { and weights (for column } \\
\text { no. } 3 \text { : } w_{1}=0.10 ; w_{2}=0.30 ; w_{2}=0.60 ; \text { for column } \\
\text { no. 4: } w_{1}=0.10 ; w_{2}=0.20 ; w_{2}=0.70 ; \text { for column } \\
\text { no. } 5: w_{1}=0.50 ; w_{2}=0.30 ; w_{2}=0.20 \text { ) }\end{array}$ & $\begin{array}{l}1730.365 \\
1776.246 \\
1822.088 \\
1867.347 \\
1912.843\end{array}$ & $\begin{array}{l}1208.646 \\
1210.870 \\
1213.481 \\
1215.922 \\
1218.376\end{array}$ & $\begin{array}{l}470.570 \\
500.931 \\
533.670 \\
548.722 \\
576.735\end{array}$ \\
\hline $10 a$ & $\begin{array}{l}\text { Simple single exponential smoothing (Brown's } \\
\text { model), } \alpha \text { opt. for min. value }{ }^{*} \Psi\end{array}$ & $\begin{array}{l}1727.833 \\
1756.928 \\
1779.589 \\
1797.239 \\
1810.987\end{array}$ & $\begin{array}{l}1210.222 \\
1212.031 \\
1213.459 \\
1214.585 \\
1215.473\end{array}$ & $\begin{array}{l}466.125 \\
460.777 \\
458.367 \\
457.281 \\
456.792\end{array}$ \\
\hline
\end{tabular}


Table 1 (continued)

Forecasts of the world steel production

\begin{tabular}{|c|c|c|c|c|}
\hline No. & Method & $\begin{array}{c}\text { Total } \\
\text { (Mt) }\end{array}$ & $\begin{array}{l}\text { BOF } \\
\text { (Mt) }\end{array}$ & $\begin{array}{l}\text { EAF } \\
\text { (Mt) }\end{array}$ \\
\hline $10 b$ & $\begin{array}{l}\text { Simple single exponential smoothing (Brown's } \\
\text { model), } \alpha \text { opt. for min. value *RMSE }\end{array}$ & $\begin{array}{l}1707.836 \\
1718.729 \\
1725.565 \\
1729.855 \\
1732.547\end{array}$ & $\begin{array}{l}1209.308 \\
1210.531 \\
1211.414 \\
1212.050 \\
1212.509\end{array}$ & $\begin{array}{l}465.195 \\
459.094 \\
456.395 \\
455.201 \\
454.673\end{array}$ \\
\hline $11_{\mathrm{a} 1}$ & $\begin{array}{l}\text { Exponential autoregressive model for k-point } \\
\qquad(k=3) \text { and } I \text {-point }(I=2) \text { and } \alpha \text { opt. for min. } \\
\text { value }{ }^{*} \Psi ; \beta_{1}=0.7 ; \quad \beta_{2}=0.2 ; \beta_{3}=0.1 ; \delta_{1}=0.2 ; \\
\delta_{2}=0.8\end{array}$ & $\begin{array}{l}1702.327 \\
1737.649 \\
1737.980 \\
1770.564 \\
1775.161\end{array}$ & $\begin{array}{l}1210.701 \\
1214.230 \\
1217.296 \\
1219.736 \\
1221.858\end{array}$ & $\begin{array}{l}468.087 \\
445.203 \\
437.374 \\
434.773 \\
435.332\end{array}$ \\
\hline $111_{\mathrm{a} 2}$ & $\begin{array}{l}\text { Exponential autoregressive model for k-point } \\
(k=3) \text { and } I \text {-point }(I=2) \text { and } \alpha \text { opt. for min. } \\
\text { value *RMSE; } \beta_{1}=0.7 ; \beta_{2}=0.2 ; \beta_{3}=0.1 ; \delta_{1}=0.2 \text {; } \\
\qquad \delta_{2}=0.8\end{array}$ & $\begin{array}{l}1689.463 \\
1707.054 \\
1688.045 \\
1707.281 \\
1698.021\end{array}$ & $\begin{array}{l}1210.153 \\
1211.143 \\
1212.428 \\
1213.233 \\
1213.916\end{array}$ & $\begin{array}{l}467.173 \\
443.875 \\
436.084 \\
433.611 \\
434.246\end{array}$ \\
\hline $12 \mathrm{~b} 1$ & $\begin{array}{c}\text { Exponential autoregressive model for k-point } \\
(k=3) \text { and I-point }(I=2) \text { and } \alpha \text { opt. for min. } \\
\text { value * } \Psi ; \text { column no. } 3: \beta_{1}=0.7 ; \quad \beta_{2}=0.2 ; \\
\beta_{3}=0.1 ; \delta_{1}=0.8 ; \delta_{2}=0.2 ; \text { columns no. 4-5: } \beta_{1}=0.5 ; \\
\quad \beta_{2}=0.3 ; \beta_{3}=0.2 ; \delta_{1}=0.8 ; \delta_{2}=0.2\end{array}$ & $\begin{array}{l}1732.610 \\
1804.755 \\
1885.117 \\
2005.275 \\
2156.854\end{array}$ & $\begin{array}{l}1211.453 \\
1214.759 \\
1217.630 \\
1219.561 \\
1221.257\end{array}$ & $\begin{array}{l}472.756 \\
454.299 \\
441.318 \\
435.408 \\
436.924\end{array}$ \\
\hline $12 \mathrm{~b} 2$ & $\begin{array}{c}\text { Exponential autoregressive model for k-point } \\
(k=3) \text { and I-point }(I=2) \text { and } \alpha \text { opt. for min. } \\
\text { value *RMSE; column no. } 3: \beta_{1}=0.7 ; \quad \beta_{2}=0.2 ; \\
\beta_{3}=0.1 ; \delta_{1}=0.8 ; \delta_{2}=0.2 ; \text { columns no. } 4-5: \beta_{1}=0.5 ; \\
\quad \beta_{2}=0.3 ; \beta_{3}=0.2 ; \delta_{1}=0.8 ; \delta_{2}=0.2\end{array}$ & $\begin{array}{l}1710.050 \\
1735.307 \\
1749.405 \\
1781.783 \\
1809.211\end{array}$ & $\begin{array}{l}1211.264 \\
1212.262 \\
1213.434 \\
1213.918 \\
1214.524\end{array}$ & $\begin{array}{l}471.343 \\
451.945 \\
438.917 \\
433.316 \\
435.084\end{array}$ \\
\hline $13 \mathrm{a} 1$ & $\begin{array}{l}\text { Exponential autoregressive model for k-point } \\
\qquad(k=2) \text { and I-point }(I=2) \text { and } \alpha \text { opt. for min. } \\
\text { value }{ }^{*} \Psi ; \beta_{1}=0.7 ; \quad \beta_{2}=0.3 ; \delta_{1}=0.8 ; \delta_{2}=0.2\end{array}$ & $\begin{array}{l}1731.419 \\
1758.892 \\
1782.800 \\
1806.388 \\
1827.015\end{array}$ & $\begin{array}{l}1209.972 \\
1213.900 \\
1216.613 \\
1219.041 \\
1221.162\end{array}$ & $\begin{array}{l}467.374 \\
446.222 \\
436.869 \\
437.219 \\
437.366\end{array}$ \\
\hline $13 \mathrm{a} 2$ & $\begin{array}{l}\text { Exponential autoregressive model for k-point } \\
\quad(k=2) \text { and I-point }(I=2) \text { and } \alpha_{\text {opt. }} \text { for min. } \\
\text { value *RMSE; } \beta_{1}=0.7 ; \quad \beta_{2}=0.3 ; \delta_{1}=0.8 ; \delta_{2}=0.2\end{array}$ & $\begin{array}{l}1707.118 \\
1697.032 \\
1691.535 \\
1693.322 \\
1694.511\end{array}$ & $\begin{array}{l}1209.149 \\
1211.400 \\
1212.509 \\
1213.520 \\
1214.290\end{array}$ & $\begin{array}{l}466.208 \\
444.573 \\
435.260 \\
435.719 \\
435.912\end{array}$ \\
\hline $14 \mathrm{~b} 1$ & $\begin{array}{l}\text { Exponential autoregressive model for k-point } \\
(k=2) \text { and I-point }(I=2) \text { and } \alpha \text { opt. for min. } \\
\text { value }{ }^{*} \Psi ; \beta_{1}=0.3 ; \quad \beta_{2}=0.7 ; \delta_{1}=0.2 ; \delta_{2}=0.8\end{array}$ & $\begin{array}{l}1701.872 \\
1725.536 \\
1718.794 \\
1729.058 \\
1733.333\end{array}$ & $\begin{array}{l}1210.145 \\
1214.459 \\
1216.418 \\
1218.327 \\
1219.891\end{array}$ & $\begin{array}{l}475.232 \\
468.157 \\
447.300 \\
448.348 \\
448.253\end{array}$ \\
\hline $14 \mathrm{~b} 2$ & $\begin{array}{l}\text { Exponential autoregressive model for } k \text {-point }(k \\
=2) \text { and I-point }(I=2) \text { and } \alpha \text { opt. for min. } \\
\text { value }{ }^{*} \text { RMSE; } \beta_{1}=0.3 ; \quad \beta_{2}=0.7 ; \delta_{1}=0.2 ; \delta_{2}=0.8\end{array}$ & $\begin{array}{l}1694.918 \\
1708.717 \\
1691.944 \\
1696.505 \\
1697.197\end{array}$ & $\begin{array}{l}1209.829 \\
1212.328 \\
1212.562 \\
1213.409 \\
1214.022\end{array}$ & $\begin{array}{l}467.115 \\
454.894 \\
432.388 \\
435.464 \\
435.762\end{array}$ \\
\hline $15 \mathrm{a} 1$ & $\begin{array}{l}\text { Holt's linear trend model with additive trend for } \\
\text { start point } S_{1}=y_{2}-y_{1} \text { and } \alpha_{\text {opt. }} \text { for min. value }{ }^{*} \Psi\end{array}$ & $\begin{array}{l}1731.578 \\
1772.771 \\
1813.963 \\
1855.156 \\
1896.348\end{array}$ & $\begin{array}{l}1210.538 \\
1214.134 \\
1217.730 \\
1221.325 \\
1224.921\end{array}$ & $\begin{array}{l}474.231 \\
476.695 \\
479.159 \\
481.623 \\
484.087\end{array}$ \\
\hline $15 \mathrm{a} 2$ & $\begin{array}{c}\text { Holt's linear trend model with additive trend for } \\
\text { start point: } S_{1}=y_{2}-y_{1} \text { and } \alpha \text { opt. for min. } \\
\text { value }{ }^{*} \mathrm{RMSE}\end{array}$ & $\begin{array}{l}1727.270 \\
1764.484 \\
1801.697 \\
1838.910 \\
1876.123\end{array}$ & $\begin{array}{l}1229.149 \\
1251.331 \\
1273.513 \\
1295.695 \\
1317.878\end{array}$ & $\begin{array}{l}474.516 \\
479.063 \\
483.610 \\
488.158 \\
492.705\end{array}$ \\
\hline $16_{\mathrm{a} 1}$ & $\begin{array}{l}\text { Holt's linear trend model with additive trend for } \\
\text { start point: } S_{1}=0 \text { and } \alpha \text { opt. for min. value }{ }^{*} \Psi\end{array}$ & $\begin{array}{l}1731.569 \\
1772.744 \\
1813.919 \\
1855.094 \\
1896.268\end{array}$ & $\begin{array}{l}1230.218 \\
1221.245 \\
1212.272 \\
1203.299 \\
1194.326\end{array}$ & $\begin{array}{l}473.630 \\
475.493 \\
477.356 \\
479.219 \\
481.082\end{array}$ \\
\hline
\end{tabular}


Table 1 (continued)

Forecasts of the world steel production

\begin{tabular}{|c|c|c|c|c|}
\hline No. & Method & $\begin{array}{l}\text { Total } \\
\text { (Mt) }\end{array}$ & $\begin{array}{l}\text { BOF } \\
\text { (Mt) }\end{array}$ & $\begin{array}{l}\text { EAF } \\
(\mathrm{Mt})\end{array}$ \\
\hline $16 \mathrm{a} 2$ & $\begin{array}{c}\text { Holt's linear trend model with additive trend for } \\
\text { start point: } S_{1}=0 \text { and } \alpha \text { opt. for min. } \\
\text { value *RMSE }\end{array}$ & $\begin{array}{l}1727.819 \\
1765.165 \\
1802.510 \\
1839.856 \\
1877.202\end{array}$ & $\begin{array}{l}1224.821 \\
1242.675 \\
1260.530 \\
1278.385 \\
1296.239\end{array}$ & $\begin{array}{l}471.395 \\
475.453 \\
479.511 \\
483.570 \\
487.628\end{array}$ \\
\hline $17 \mathrm{a} 1$ & $\begin{array}{c}\text { Holt's linear trend model with multiplicative trend } \\
\text { for start point } S_{1}=y_{2} / y_{1} \text { and } \alpha \text { opt. for min. } \\
\text { value }{ }^{*} \Psi\end{array}$ & $\begin{array}{l}1721.978 \\
1754.418 \\
1787.470 \\
1821.144 \\
1855.453\end{array}$ & $\begin{array}{l}1225.999 \\
1245.332 \\
1264.970 \\
1284.918 \\
1305.181\end{array}$ & $\begin{array}{l}474.486 \\
477.221 \\
479.972 \\
482.738 \\
485.520\end{array}$ \\
\hline $17 \mathrm{a} 2$ & $\begin{array}{l}\text { Holt's linear trend model with multiplicative trend } \\
\text { for start point: } S_{1}=y_{2} / y_{1} \text { and } \alpha \text { opt. for min. } \\
\text { value * RMSE }\end{array}$ & $\begin{array}{l}1735.098 \\
1780.900 \\
1827.911 \\
1876.163 \\
1925.688\end{array}$ & $\begin{array}{l}1245.615 \\
1285.497 \\
1326.657 \\
1369.134 \\
1412.972\end{array}$ & $\begin{array}{l}476.685 \\
482.409 \\
488.202 \\
494.064 \\
499.997\end{array}$ \\
\hline $18 \mathrm{a} 1$ & $\begin{array}{l}\text { Holt's linear trend model with multiplicative trend } \\
\text { for start point: } S_{1}=1 \text { and } \alpha \text { opt. for min. value }{ }^{*} \Psi\end{array}$ & $\begin{array}{l}1728.517 \\
1767.612 \\
1807.535 \\
1848.360 \\
1890.106\end{array}$ & $\begin{array}{l}1241.151 \\
1276.302 \\
1312.448 \\
1349.618 \\
1387.840\end{array}$ & $\begin{array}{l}473.717 \\
475.674 \\
477.640 \\
479.613 \\
481.595\end{array}$ \\
\hline $18 \mathrm{a} 2$ & $\begin{array}{c}\text { Holt's linear trend model with multiplicative trend } \\
\text { for start point: } S_{1}=1 \text { and } \alpha \text { opt. for min. } \\
\text { value *RMSE }\end{array}$ & $\begin{array}{l}1735.570 \\
1781.866 \\
1829.397 \\
1878.196 \\
1928.296\end{array}$ & $\begin{array}{l}1246.615 \\
1287.561 \\
1329.853 \\
1373.533 \\
1418.649\end{array}$ & $\begin{array}{l}472.925 \\
477.865 \\
482.856 \\
487.898 \\
492.994\end{array}$ \\
\hline $19_{\mathrm{a} 1}$ & $\begin{array}{l}\text { Holt's linear trend model with additive damped } \\
\text { trend for start point: } S_{1}=y_{2}-y_{1} \text { and } \alpha \text { opt. for min. } \\
\qquad \text { value } \Psi\end{array}$ & $\begin{array}{l}1721.800 \\
1712.477 \\
1712.465 \\
1705.616 \\
1699.809\end{array}$ & $\begin{array}{l}1210.588 \\
1214.214 \\
1217.838 \\
1221.461 \\
1225.082\end{array}$ & $\begin{array}{l}493.651 \\
489.518 \\
482.553 \\
477.581 \\
474.693\end{array}$ \\
\hline $199_{2}$ & $\begin{array}{l}\text { Holt's linear trend model with additive damped } \\
\text { trend for start point: } S_{1}=y_{2}-y_{1} \text { and } \alpha \text { opt. for min. } \\
\text { value *RMSE }\end{array}$ & $\begin{array}{l}1726.022 \\
1761.071 \\
1795.627 \\
1829.695 \\
1863.281\end{array}$ & $\begin{array}{l}1216.221 \\
1223.964 \\
1230.363 \\
1235.587 \\
1239.786\end{array}$ & $\begin{array}{l}471.688 \\
471.688 \\
471.688 \\
471.688 \\
471.688\end{array}$ \\
\hline $20 \mathrm{a} 1$ & $\begin{array}{l}\text { Holt's linear trend model with additive damped } \\
\text { trend for start point: } S_{1}=0 \text { and } \alpha \text { opt. for min. } \\
\qquad \text { value }^{\star} \Psi\end{array}$ & $\begin{array}{l}1721.800 \\
1721.477 \\
1713.465 \\
1705.616 \\
1699.809\end{array}$ & $\begin{array}{l}1210.243 \\
1213.115 \\
1215.603 \\
1217.743 \\
1219.517\end{array}$ & $\begin{array}{l}471.684 \\
471.684 \\
471.684 \\
471.684 \\
471.684\end{array}$ \\
\hline $20_{\mathrm{a} 2}$ & $\begin{array}{l}\text { Holt's linear trend model with additive damped } \\
\text { trend for start point: } S_{1}=0 \text { and } \alpha \text { opt. for min. } \\
\text { value *RMSE }\end{array}$ & $\begin{array}{l}1722.856 \\
1754.690 \\
1785.365 \\
1814.924 \\
1843.385\end{array}$ & $\begin{array}{l}1213.062 \\
1217.829 \\
1221.462 \\
1224.156 \\
1226.073\end{array}$ & $\begin{array}{l}471.684 \\
471.684 \\
471.684 \\
471.684 \\
471.684\end{array}$ \\
\hline $21 \mathrm{a} 1$ & $\begin{array}{l}\text { Holt's linear trend model with multiplicative } \\
\text { damped trend for start point: } S_{1}=\mathrm{y}_{2} / \mathrm{y}_{1} \text { and } \\
\qquad \alpha \text { opt. for min. value }{ }^{\star} \Psi\end{array}$ & $\begin{array}{l}1717.175 \\
1744.691 \\
1772.648 \\
1801.054 \\
1829.914\end{array}$ & $\begin{array}{l}1206.971 \\
1206.981 \\
1206.991 \\
1207.007 \\
1207.012\end{array}$ & $\begin{array}{l}473.094 \\
474.425 \\
475.760 \\
477.098 \\
478.440\end{array}$ \\
\hline $21_{\mathrm{a} 2}$ & $\begin{array}{l}\text { Holt's linear trend model with multiplicative } \\
\text { damped trend for start point: } S_{1}=\mathrm{y}_{2} / \mathrm{y}_{1} \text { and } \\
\qquad \alpha_{\text {opt. }} \text { for min. value*RMSE }\end{array}$ & $\begin{array}{l}1731.007 \\
1772.776 \\
1815.553 \\
1859.361 \\
1904.227\end{array}$ & $\begin{array}{l}1212.224 \\
1217.506 \\
1222.812 \\
1228.141 \\
1233.493\end{array}$ & $\begin{array}{l}476.102 \\
480.477 \\
484.892 \\
489.347 \\
493.843\end{array}$ \\
\hline
\end{tabular}


Table 1 (continued)

Forecasts of the world steel production

\begin{tabular}{|c|c|c|c|c|}
\hline No. & Method & $\begin{array}{c}\text { Total } \\
\text { (Mt) }\end{array}$ & $\begin{array}{l}\text { BOF } \\
\text { (Mt) }\end{array}$ & $\begin{array}{l}\text { EAF } \\
\text { (Mt) }\end{array}$ \\
\hline $22 \mathrm{a} 1$ & $\begin{array}{c}\text { Holt's linear trend model with multiplicative } \\
\text { damped trend for start point: } S_{1}=1 \text { and } \alpha \text { opt. for } \\
\text { min. value } \Psi\end{array}$ & $\begin{array}{l}1727.874 \\
1766.196 \\
1805.369 \\
1845.411 \\
1886.340\end{array}$ & $\begin{array}{l}1209.653 \\
1212.350 \\
1215.054 \\
1217.764 \\
1220.480\end{array}$ & $\begin{array}{l}471.700 \\
472.856 \\
474.015 \\
475.177 \\
476.342\end{array}$ \\
\hline $22 \mathrm{a} 2$ & $\begin{array}{c}\text { Holt's linear trend model with multiplicative } \\
\text { damped trend for start point: } S_{1}=1 \text { and } \alpha \text { opt. for } \\
\text { min. value }{ }^{*} \text { RMSE }\end{array}$ & $\begin{array}{l}1731.697 \\
1774.184 \\
1871.714 \\
1862.311 \\
1908.003\end{array}$ & $\begin{array}{l}1208.708 \\
1210.455 \\
1212.205 \\
1213.957 \\
1215.712 \\
\end{array}$ & $\begin{array}{l}473.880 \\
477.195 \\
480.534 \\
483.896 \\
487.281\end{array}$ \\
\hline $23 \mathrm{a} 1$ & $\begin{array}{l}\text { Holt's quadratic trend model with additive } \\
\text { formula for point start: } S_{1}=\mathrm{y}_{2}-\mathrm{y}_{1} \text { and } \alpha_{\text {opt. }} \text { for } \\
\text { min. value } \Psi\end{array}$ & $\begin{array}{l}1862.290 \\
1915.889 \\
1965.536 \\
2023.232 \\
2076.976\end{array}$ & $\begin{array}{l}1210.250 \\
1209.632 \\
1205.106 \\
1196.673 \\
1184.332\end{array}$ & $\begin{array}{l}440.563 \\
443.369 \\
449.546 \\
459.094 \\
472.014\end{array}$ \\
\hline $23 \mathrm{a} 2$ & $\begin{array}{l}\text { Holt's quadratic trend model with additive } \\
\text { formula for point start: } S_{1}=\mathrm{y}_{2}-\mathrm{y}_{1} \text { and } \alpha \text { opt. for } \\
\text { min. value }{ }^{*} \mathrm{RMSE}\end{array}$ & $\begin{array}{l}1806.171 \\
1856.092 \\
1906.009 \\
1955.921 \\
2005.828\end{array}$ & $\begin{array}{l}1235.728 \\
1263.626 \\
1290.661 \\
1316.833 \\
1342.142\end{array}$ & $\begin{array}{l}441.989 \\
444.883 \\
449.850 \\
456.890 \\
466.002\end{array}$ \\
\hline $24 \mathrm{a} 1$ & $\begin{array}{c}\text { Holt's quadratic trend model with additive } \\
\text { formula for point start: } S_{1}=0 \text { and } \alpha \text { opt. for min. } \\
\text { value } \Psi\end{array}$ & $\begin{array}{l}1906.375 \\
1966.167 \\
2025.940 \\
2085.693 \\
2145.427\end{array}$ & $\begin{array}{l}1209.082 \\
1207.651 \\
1202.666 \\
1194.127 \\
1182.035\end{array}$ & $\begin{array}{l}451.938 \\
456.386 \\
461.005 \\
465.945 \\
471.057\end{array}$ \\
\hline $24 \mathrm{a} 2$ & $\begin{array}{c}\text { Holt's quadratic trend model with additive } \\
\text { formula for point start: } S_{1}=0 \text { and } \alpha \text { opt. for min. } \\
\text { value *RMSE }\end{array}$ & $\begin{array}{l}1834.603 \\
1889.946 \\
1945.358 \\
2000.838 \\
2056.386\end{array}$ & $\begin{array}{l}1232.951 \\
1255.747 \\
1275.352 \\
1291.768 \\
1304.995\end{array}$ & $\begin{array}{l}446.885 \\
450.565 \\
454.691 \\
459.263 \\
464.280\end{array}$ \\
\hline $25 \mathrm{a} 1$ & $\begin{array}{l}\text { Brown's double exponential smoothing (linear) } \\
\qquad \text { and } \alpha \text { opt. for min. value }{ }^{\star} \Psi\end{array}$ & $\begin{array}{l}1706.754 \\
1732.993 \\
1759.231 \\
1785.470 \\
1811.709\end{array}$ & $\begin{array}{l}1253.150 \\
1272.900 \\
1292.649 \\
1312.650 \\
1332.149\end{array}$ & $\begin{array}{l}453.604 \\
460.093 \\
466.582 \\
473.071 \\
479.560\end{array}$ \\
\hline $25 \mathrm{a} 2$ & $\begin{array}{l}\text { Brown's double exponential smoothing (linear) } \\
\text { and } \alpha \text { opt. for min. value *RMSE }\end{array}$ & $\begin{array}{l}1706.917 \\
1733.236 \\
1759.555 \\
1785.875 \\
1812.194\end{array}$ & $\begin{array}{l}1209.897 \\
1213.054 \\
1216.211 \\
1219.368 \\
1222.525\end{array}$ & $\begin{array}{l}453.117 \\
459.457 \\
465.797 \\
472.137 \\
478.478\end{array}$ \\
\hline $266_{a 1}$ & $\begin{array}{l}\text { Brown's triple exponential smoothing (quadratic) } \\
\qquad \text { and } \alpha \text { opt. for min. value }{ }^{\star} \Psi\end{array}$ & $\begin{array}{l}1695.716 \\
1709.094 \\
1722.472 \\
1735.850 \\
1749.229\end{array}$ & $\begin{array}{l}1193.375 \\
1178.536 \\
1163.697 \\
1148.858 \\
1134.019\end{array}$ & $\begin{array}{l}452.873 \\
459.014 \\
465.155 \\
471.295 \\
477.436\end{array}$ \\
\hline $26_{\mathrm{a} 2}$ & $\begin{array}{l}\text { Brown's triple exponential smoothing (quadratic) } \\
\text { and } \alpha \text { opt. for min. value *RMSE }\end{array}$ & $\begin{array}{l}1706.909 \\
1720.830 \\
1734.056 \\
1747.630 \\
1761.203\end{array}$ & $\begin{array}{l}1201.397 \\
1184.734 \\
1168.071 \\
1151.408 \\
1134.746\end{array}$ & $\begin{array}{l}452.805 \\
458.221 \\
463.636 \\
469.051 \\
474.467\end{array}$ \\
\hline $27 \mathrm{a} 1$ & $\begin{array}{l}\text { Advanced exponential autoregressive model } \\
\text { and } \alpha \text { opt. for min. value }{ }^{\star} \Psi\end{array}$ & $\begin{array}{l}1733.301 \\
1776.133 \\
1818.965 \\
1861.797 \\
1904.630\end{array}$ & $\begin{array}{l}1210.716 \\
1214.531 \\
1218.345 \\
1222.159 \\
1225.974\end{array}$ & $\begin{array}{l}459.461 \\
475.009 \\
490.557 \\
506.104 \\
521.652\end{array}$ \\
\hline
\end{tabular}


Table 1 (continued)

Forecasts of the world steel production

\begin{tabular}{|c|c|c|c|c|}
\hline No. & Method & $\begin{array}{l}\text { Total } \\
(\mathrm{Mt})\end{array}$ & $\begin{array}{l}\text { BOF } \\
(\mathrm{Mt})\end{array}$ & $\begin{array}{l}\text { EAF } \\
\text { (Mt) }\end{array}$ \\
\hline $27 \mathrm{a} 2$ & $\begin{array}{l}\text { Advanced exponential autoregressive model } \\
\text { and } \alpha \text { opt. for min. value *RMSE }\end{array}$ & $\begin{array}{l}1717.326 \\
1755.847 \\
1796.368 \\
1836.890 \\
1877.411\end{array}$ & $\begin{array}{l}1210.751 \\
1215.609 \\
1220.466 \\
1225.324 \\
1230.182\end{array}$ & $\begin{array}{l}457.382 \\
472.135 \\
486.887 \\
501.640 \\
516.392\end{array}$ \\
\hline 28. & Creep trend and harmonic weights method & $\begin{array}{l}1723.048 \\
1755.618 \\
1788.187 \\
1820.187 \\
1853.326 \\
\end{array}$ & $\begin{array}{l}1232.629 \\
1258.295 \\
1283.960 \\
1309.626 \\
1335.292\end{array}$ & $\begin{array}{l}479.450 \\
487.122 \\
494.794 \\
502.465 \\
510.137\end{array}$ \\
\hline 29. & Linear model & $\begin{array}{l}1829.988 \\
1884.130 \\
1938.272 \\
1992.414 \\
2046.556\end{array}$ & $\begin{array}{l}1354.003 \\
1401.010 \\
1448.017 \\
1495.024 \\
1542.031\end{array}$ & $\begin{array}{l}472.974 \\
482.144 \\
491.314 \\
500.484 \\
509.655\end{array}$ \\
\hline 30. & Logarithmic model & $\begin{array}{l}1831.222 \\
1885.104 \\
1938.960 \\
1992.789 \\
2046.591\end{array}$ & $\begin{array}{l}1355.019 \\
1401.797 \\
1448.553 \\
1495.286 \\
1541.995\end{array}$ & $\begin{array}{l}474.321 \\
483.449 \\
492.573 \\
501.693 \\
510.808\end{array}$ \\
\hline
\end{tabular}

Information about forecast period: a) in 2018 year, $b$-e) in 2019-2022 for all columns in Table 1.

${ }^{*} \Psi$ and ${ }^{*} R M S E$ - forecast errors.

The best models (analysis of forecast errors and $R 2$ for models no. 29-30) were presented on the Figure 2 (point: 3.2).

Source: (Gajdzik, 2018).

\section{Analysis of trends of obtained forecasts for the world steel production}

Analyzing the trends of obtained global steel production forecasts (Figure 2), a projected upward tendency is observed. In the optimistic scenario, it can be assumed that steel production in the world in 2022 will exceed 2000 million tonnes (Mt in Table 1). BOF's share (as before) is larger than EAF. The forecasted BOF steel production is growing faster than the predicted EAF steel production. In an optimistic scenario, it may exceed 1500 million tonnes (Mt in Table 1) in 2022.

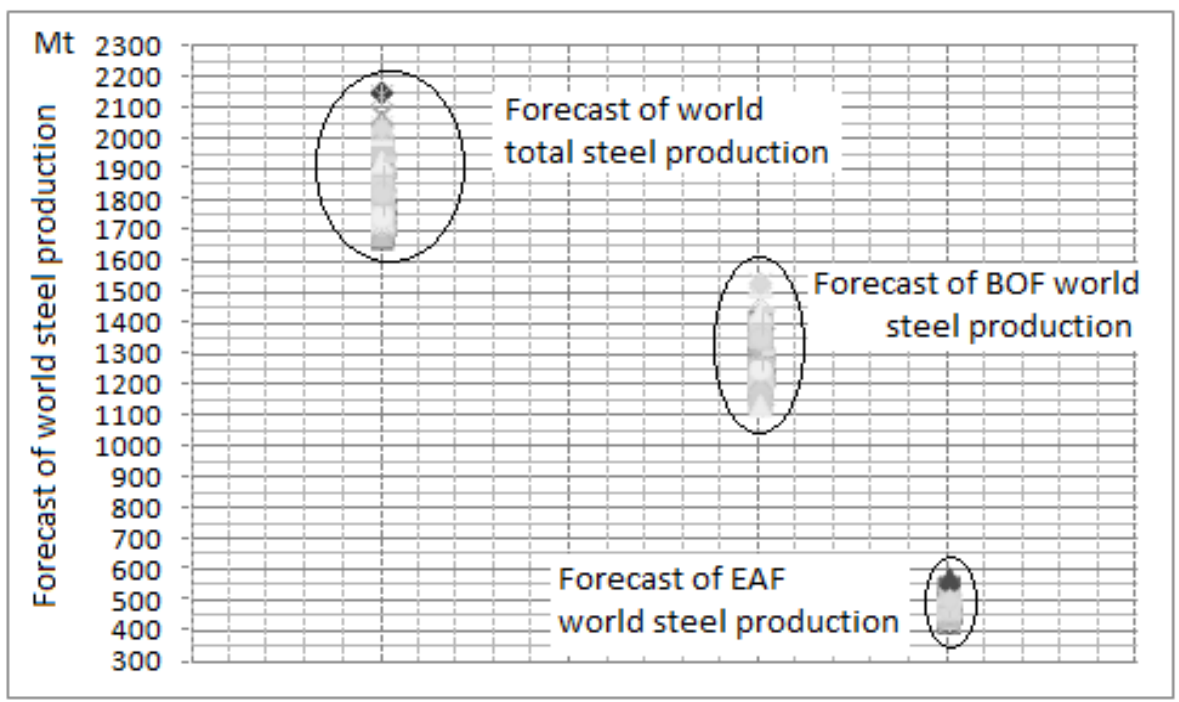

Fig. 2 Forecasts of world steel production 


\section{CONCLUSION}

The implementation of technology required by Industry 4.0 in steel enterprises (metallurgical market) is implemented gradually, and current investments mainly concern on production automation. Recording production data, which has been extended in steel mills (along with the development of IT), facilitates the analysis of steel production using predictions. The projection of the forecasts presented in the publication (Gajdzik, 2018) indicated growing trends in the volume of steel production in the world, both in terms of forecasting which was total steel production and the following ranges: BOF steel production and EAF steel production.

\section{REFEENCES}

Bauernhansl, T., Ten Hompel, M. and Vogel-Henser, B. (2014). Industrie 4.0 in Produkten. Automatisierung und Logistik. Wiesbaden: Springer Fachmedien.

Dittmann, P. (2011). Prognozowanie w zarządzaniu sprzedażą i finansami przedsiębiorstwa. Warszawa: Oficyna a Wolters Kluwer business.

Dittmann, P. (2016). Prognozowanie w przedsiębiorstwie. Metody i ich zastosowanie. Kraków: Wydawnictwo Nieoczywiste.

Furman, J., Kuczyńska-Chałada, M., Pawlak, S. and Grabowska, S. (2017). The influence of Lean Manufacturing tools on the product quality in the casting process - case study. In: METAL 2017: 26th Anniversary International Conference on Metallurgy and Materials, Ostrava: Tanger, pp. 2127.

Gajdzik, B. (2018). Models of production function for the steel industry after restructuring process with forecasts and scenarios of changes in volume of steel production. Gliwice: The Silesian University of Technology.

Gajdzik, B. (2017). Prognostic modeling of total global steel production. Metalurgija 1-2 (56), pp. 279- 282.

Gajdzik, B. (2013). The road of Polish steelworks towards market success - changes after restructuring process. Metalurgija, 3, pp. 421-424.

Gajdzik, B. (2019). Visions and directions for the development of logistics 4.0 in context 4.0 industrial revolution (level 4.0 - L.4.0). In: Production management and packaging. Food safety and industry 4.0, ed. A. Walaszczyk, I. Jałmużna and 1J. Lewandowski. Łódź: Wydaw. Politechniki Łódzkiej, pp. 69-79. Monografie Politechniki Łódzkiej, 2305.

Available at : http://cybra.lodz.pl/dlibra/docmetadata?id=16169\&from=publication

Gajdzik, B. and Gawlik, R. (2018) Choosing the production function model for an optimal measurement of the restructuring efficiency of the Polish metallurgical sector in years 2000-2015. Metals, 8(23), pp. 2-11.

Gajdzik, B. and Sitko, J. (2014). An analysis of the causes of complaints about steel sheets in metallurgical products quality management systems. Metalurgija, 1 (53), JanuaryMarch, pp. 135-138.

Gajdzik, B. and Sitko, J. (2016). Steel mill product analysis using quality methods. Metalurgija, 4 (55), pp. 807-810.

Gajdzik, B. and Sroka, W. (2012). Analytic study of the capital restructuring process in metallurgical enterprises around the World and in Poland. Metalurgija 2 (51), pp. 265268.

Gerbert, P., Lorenz, M., Rüßmann, M., Waldner, M., Justus, J., Engel, P. and Harnisch, M. Industry 4.0: The Future of Productivity and Growth in Manufacturing Industries [online].Available at: www.bcg.com. [Accessed: 9 Apr.2015].

Grabowska, S. (2016). Business model metallurgical company built on the competitive advantage. METAL 2016. 25th Anniversary International Conference on Metallurgy and Materials, May 25th-27th, 2016. Brno, Czech Republic. Ostrava: Tanger Ltd., pp. 18001807.

Grabowska, S. (2018). Implement of the Heat Treatment Process in the Industry 4.0 Context, in 27th International Conference on Metallurgy and Materials. Metal 2018. Brno: Tanger Ltd., pp. 1985-1990.

Green, W.H. (2003). Econometric Analysis. 5 ed. Upper Saddle River, NJ, Prentice Hall. 
Jasperneite, J. (2012). Was hinter Begriffen wie Industrie 4.0 steckt. Computer \& Automation. 19 Dec.2012 [Accessed 27 Jan. 2016].

Kagermann, H., Wahlster W. and Helbig J., eds. (2013). Recommendations for implementing the strategic initiative Industrie 4.0: Final report of the Industrie 4.0 Working Group.

Kramarz, M. (2012). Strategie adaptacyjne przedsiębiorstw flagowych sieci dystrybucji z odroczoną produkcją: dystrybucja wyrobów hutniczych. Gliwice: Wydawnictwo Politechniki Śląskiej.

Report PWC: Przemysł 4.0 czyli wyzwania współczesnej produkcji. [online] Available at: https://www.pwc.pl/pl/pdf/przemysl-4-0-raport.pdf [Accessed: 20 May 2018].

Saniuk, A., Witkowski, K. and Saniuk, S. (2013). Management of production orders in metalworking production / // W: 22nd International Conference on Metallurgy and Materials - METAL 2013. Brno: Tanger Ltd., pp. 2057-2062.

Schwab, K. (2016). The Fourth Industrial Revolution [Accessed 27 Jan. 2016].

Sendler, U. (2013). Industrie 4.0 - Die Beherrschung der industrieller Komplexität mit SysLM. Berlin: Springer Vieweg.

Sitko, J. (2015). The intelligent process of initiating new product in aspect problems of management. 15th International Multidisciplinary Scientific GeoConference SGEM 2015. Ecology, economics, education and legislation, 18-24, June, 2015, Albena, Bulgaria. Conference proceedings. Vol. 3, Environmental Economics, Education \& Accreditation in Geosciences. Sofia: STEF92 Technology, pp. 689-696.

Sitko, J. Mikus, R., Bożek, P. (2018) Analysis of device failure in the mechanical production plant. MAPE 2018. XV International Conference Multidisciplinary Aspects of Production Engineering, 05-08 September 2018, Zawiercie, Poland. Conference proceedings, 1(1), pp. 93-99.

Snarska, A. (2005). Statistics, Econometrics, Prognosis. Warsaw: Placet.

Sroka, W., Cygler, J., Gajdzik, B. (2014). Knowledge transfer in networks - the case of steel enterprises in Poland. Metalurgija, 1 (53), January-March, pp. 101-104.

StatSoft (2012). Prediction in business. Cracow: StatSoft.

Steel Statistical Yearbook. World Steel Association.[online] Available at:

https://www.worldsteel.org/internet-2017/steel-by-topic/statistics/steel-statistical-yearbook.html] and other reports from 2016 to 2001.

Time for 4.0. Special Report. (2018). Newsletter [Accessed 26 Feb. 2019]. Automatyka 10/2018. [online] Available at: wnp.pl, https://automatykaonline.pl/Artykuly/Przemysl4.0/Czas-na-cztery-zero.-Raport-specjalny-2018].

World Steel Association report: Top steelmakers in 2017.

Zeliaś, A. (1997). Theory of forecast. Warsaw: PWE. 
Abstract. This paper presents the importance of the prediction of steel production in industry 4.0 along with forecasts for steel production in the world until 2022. In the last two decades, the virtual world has been increasingly entering production. Today's manufacturing systems are becoming faster and more flexible - easily adaptable to new products. Steel is the basic structural material (base material) for many industrial sectors. Industries such as automotive, mechanical engineering, construction and transport use steel in their production processes. Prediction methods in cyberphysical production systems are gaining in importance. The task of prediction is to reduce risk in the decision-making process. In autonomous manufacturing systems in industry 4.0 the role of prediction is more active than passive. Forecasts have the following functions: warning, reaction, prevention, normative, etc. The growing number of customized solutions in industry 4.0 translates into new challenges in the production process. Manufacturers must respond to individual customer needs more quickly, be able to personalize products while reducing energy and resource costs (saving energy and resources can increase the product competitiveness). The modern market becomes increasingly unpredictable. Production prediction under such conditions should be carried out continuously, which is possible because there is more empirical data and access to data. Information from the ongoing monitoring of the company's production is directly transferred to the prospective evaluation. In view of the contemporary reciprocal use of automation, data processing, data exchange and manufacturing techniques, there is greater access to external data, e.g. on production in different target markets and with global, international, national, regional coverage. Companies can forecast in real time, and the forecasts obtained give the possibility to quickly change their production. Industry 4.0 (from the business objective point of view) aims to provide companies with concrete economic benefits primarily by reducing manufacturing costs, standardizing and stabilizing quality, increasing productivity. Industry 4.0 aims to create a given autonomous smart factory system in which machines, factory components and services communicate and cooperate with each other, producing a personalized product. The aim of this paper is to present new challenges in the production processes in relation to steel production, as well as to prepare and present forecasts of (quantitative) steel production of territorial, global and temporary range until 2022, taking into account the applied production technologies (BOF and EAF). For forecasting purposes, classic trend models and adaptive trend models were used. This methodology was used to build separate forecasts for: total steel production, BOF steel and EAF steel. Empirical data is world steel production in 2000-2017 (annual production volume in Mt).

Keywords: steel production, Industry 4.0, prediction, forecasts 